\title{
Driving Complementarity in Interdisciplinary Research: A Reflection
}

\author{
Penelope Siebert, School of Social Sciences, Nottingham Trent University, UK \\ Peer-Olaf Siebers, School of Computer Science, University of Nottingham, UK \\ Elvira Perez Vallejos, School of Medicine, University of Nottingham, UK \\ Tommy Nilsson, School of Computer Science, University of Nottingham, UK
}

\section{Introduction}

It is increasingly recognised that traditional discipline-bound research is no longer a driver for advancing our knowledge. Currently, interdisciplinary research is heralded as an important way forward to achieve research outcomes, values and impact to address current issues in society (Mallegowda 2013). This movement towards an interdisciplinary approach to research is reflected in the growing number of funding bodies specifically encouraging research that is interdisciplinary in nature (Gleed and Marchant, 2016). It is important therefore to ask, what does interdisciplinary research mean in practice? Aboebela et al. (2007) note that there is no clear definition of interdisciplinary research. They argue that this leaves the notion of interdisciplinary research open to interpretation, resulting in the existence of varying degrees and conceptualisations of interdisciplinary research. This variation can be illustrated in the growing number of terms now associated with interdisciplinarity within research communities. Examples include multidisciplinary research, where researchers draw on different disciplines in an additive way to address a common problem as defined within one discipline, interdisciplinary research, where researchers integrate knowledge and methods from different disciplines, using real synthesis of approaches, and transdisciplinary research, where researchers from different disciplines work jointly to create innovations that integrate and move beyond discipline-specific approaches to address a common problem not defined in one specific discipline (van Teijlingen et al 2019).

In response, Aboebela et al (2007) put forward a definition to encapsulate the core concepts of interdisciplinary research.

\begin{abstract}
"Interdisciplinary research is any study or group of studies undertaken by scholars from two or more distinct scientific disciplines. The research is based upon a conceptual model that links or integrates theoretical frameworks from those disciplines, uses study design and methodology that is not limited to any one field, and requires the use of perspectives and skills of the involved disciplines throughout multiple phases of the research process." (Aboebela et al 2007 p341)
\end{abstract}

A good way to learn how to approach interdisciplinary research in practice is to look at positive examples. While we acknowledge that there are some good stories that provide helpful guidance (e.g. Brown et al 2015; Huutoniemi et al 2010; Goulden et al 2017; Danermark 2019), we would like to share our own experience by offering a reflective account of how it can work in practice, in keeping with Aboebela et al's definition. We do this by drawing on Kolb's experiential learning cycle (Kolb 1984) to describe our transformation from being four individual academics from contrasting disciplines, to an interdisciplinary research group. We comment on how this process positively influenced and still influences our general research capabilities and we talk about the challenges we had, in relation to achieving principles of interdisciplinary working and thinking. We draw on our experience of working together to explore how an agent-based modelling framework that was originally developed by two computer scientists (Siebers \& Klügl 2017) for modelling human centric and human-natural systems in the field of Social Simulation could be used in a healthcare context. This paper illustrates an exercise of reflective practice and our experience when applying the agent-based modelling framework in a workshop aiming to generate an understanding of the role of ethics in the area of digital mental health. 


\section{Interdisciplinary Group Formation}

Here we summarise who we are and how our interdisciplinary research group emerged over time. We are a group of researchers who were initially drawn together because we had limited understanding and knowledge of each other's specific disciplines, ontologies and epistemologies. We were curious to find out whether we had shared commonalities and if so, how we could combine these.

The group members are

- Author 1: (A1) Health and Social Care; Public Health Professional; Lecturer

- Author 2: (A2) Computer Science; Social Simulation; Assistant Professor

- Author 3: (A3) Mental Health and Digital Tech; Associate Professor

- Author 4: (A4) Design; Human Computer Interaction; PhD Student

A2 was developing and testing an agent based-modelling framework for co-creating social simulation models within the confines of the "Applied Computer Science" discipline. Agent-based modelling represents a powerful paradigm that can be used for conducting "what-if" analysis of human centric complex adaptive systems by modelling people as a collection of autonomous decision-making entities called agents. Each agent individually assesses its situation and makes decisions based on a set of predetermined rules. The individual agents then interact with each other and their environment to produce complex collective behaviour patterns, which in turn allows us to make conclusions based on the system's emergent properties (Bonabeau 2002). For more details on the above-mentioned framework, please refer to Siebers \& Klügl (2017).

So far, A2 had only worked with colleagues with the same academic mindset and research interests. At a university organised event "Sustainable and Resilient Cities Research Priority Area" A2 met A1. A1 asked questions about the framework as she felt it had potential as a tool to improve communications among professionals within healthcare settings in the area of patient safety. In addition, she could see some connections between her discipline of public health with aspects of the human centric adaptive systems. She told her colleagues in the Business School about it. A2 and A1 then got together with colleagues from the Business School and the local hospital to write a funding proposal with the aim to test the framework's applicability in supporting communication between doctors and nurses in a hospital setting, as well as creating a social simulation model of patient safety scenarios. A1 continued to promote the framework, this time to her colleague A3, an Associate Professor who was doing research in the area of digital mental health. A3 saw A2's framework as a novel approach to information gathering and wanted to see how it could be used as a tool to structure a workshop aimed at exploring the ethics of digital technology for mental health. Jointly, the group (A1-A3) agreed to test the application of an evolved version of the framework. At this point A4 joined the team with his expertise and interest in developing empathy for complex usability contexts. Over a two-year period, the extended group (A1-A4) met informally at cafés and at each other's departments, engaging in discussion about the fundamentals of each other's disciplinary approach and perspectives.

\section{Reflecting on our experiences as an interdisciplinary team}

In this section we present our reflections on our overall experiences during and beyond the project and what we learnt from the process, and we use Kolb's experiential learning cycle to guide our reflection. Kolb's experiential learning cycle represents a four stage learning approach and is based on Kolb's experiential learning theory, which purports that learning takes place through the process of reflecting on a concrete experience. In this context, learning is defined by Kolb as "the process whereby knowledge is created through the transforming experience" (Kolb 1984 p38).

\section{Individual Reflections}


In this section, each member of the group (A1-A4) shares their key experiences from the process of working together and reflects on what they think has helped to become an effective interdisciplinary team and shifted the team's thinking and research capabilities. We use the four stages of Kolb's experiential learning cycle: Concrete Experience (doing something), Reflective Observation (thinking about the experience), Abstract Conceptualisation (making generalisations), and Active Experimentation (putting what was learned into practice) to guide our reflections.

A1's goal for participation:

- To explore how to inform people about underlying issues and concerns related to digital technology (from a public health perspective).

A1's key experiences:

- I started the process knowing very little about Artificial Intelligence (AI), simulation models, and the world of Computer Science. In my discussions with A2, I used different analogies to try and make sense of this new world, the terminology of Computer Science, and how computer scientists approached scientific inquiry. A key moment of learning and understanding came when $A 2$ accepted that the only way I could conceptualise

Photo of Researcher what the framework could do was to think of it as a version of the computer game "The Sims" (Wikipedia 2019). This was a computer game my children used to play. To aid our collective understanding of the world of Computer Science and $\mathrm{Al}$, we agreed that we had to be flexible in the use of terminology. I had to rethink how I expressed ideas, to take into account that in each discipline the same things can be expressed differently; we just needed to ensure that the meanings did not get lost. With this new insight, I was able to understand what kind of information was needed to develop the simulation models and how my conceptualisation of realities could be transposed in the world of Al.

- I could see what the different disciplines - Social Science, Anthropology, and Computer Science - added to enhance our collective understanding of the ethical and social consequences of human interactions with new technologies in the healthcare context. The way we - as a group - approached writing our first paper was an illuminating experience for me. The process was approached in a very pragmatic way, with each of us focusing on what needed to be achieved and being very supportive of each other.

A2's goal for participation:

- Gather information for developing social simulation models for "what-if" analysis to ultimately inform policy makers about issues and solutions related to digital mental health.

A2's key experiences:

- I developed a framework that I thought I would own, and I only intended to offer others to apply it for their purposes. But suddenly it got taken over by others, requesting changes and interpreting things differently. Initially I said "oh no - we cannot use it like this"; "oh no - it's not meant like this". I had to learn to let go and open up and be collaborative. It is the nature of the interaction I had that triggered a shift in my thinking. I changed the way I thought about my framework, realising the naming might not be ideal for the wider group, and that people might prioritise other aspects of the framework, and that the framework can evolve into a much more versatile tool when ownership is shared.

- I could not think about running a focus group without a kind of structure, as it appears to be something "natural" in Software Engineering for the tasks of System Analysis and Design. My 
brain works in line with these kinds of structured approaches to capture information. A3 said hers does not. Something new for me was the idea that the framework helps to set and reiterate "ground rules" for the focus group, so that every voice can be heard. This was always seen as "a matter of course". Setting these ground rules implicitly allows collecting the viewpoints of all participants, without explicitly having to point that out.

- Through the discussions within the small group of collaborators, I realised some shortcomings in the way I generally see the world from a domain perspective; I broadened my horizon by listening to the group and vice versa, and with the help of these discussions the framework adapted into something applicable to multiple disciplines.

- I was surprised when I found out at my first meeting with A3 that the framework was supposed to be used for a very different purpose it was originally intended for, i.e. not for modelling but for a structured approach to communication. The same is true for the focus group outcomes, which were used for ethnographic purposes rather than for model development. I found through this experience that the framework has much more potential, which I would have most likely not been aware of without this kind of interdisciplinary research work. It was fascinating to see it being used in a new domain and for a new purpose, and being part of the experience. I also found the experience of working on a joint conference paper very interesting, as we all have different "discipline specific" approaches to writing papers. I usually write technical papers on my own or with people from the same domain, so it was interesting to see, the different kind of language that is used by people when writing about the same things, and where the focus is on when describing specific things.

A3's goal for participation:

- To identify research topics for digital mental health research.

A3's key experiences:

- I felt an initial sense of frustration when using the word 'hypothesis', 'problem' and 'research question' in focus group discussions as it had to be explained and the group had to agree on the shared definition meaning. Understanding each other's perspectives made participants aware of language issues and reflect on the aetiology of these differences in meaning. As an observer, I felt that this had an impact on how participants expressed themselves to promote shared understanding.

- The framework supported a new way to facilitate and structure focus group discussions. It provided an inclusive structured discussion that was focused with a tone that was non-confrontational. Disagreements did not have to be resolved and there were no opportunities for judgemental discussions, which was very refreshing and somehow unusual within academic settings. All opinions were treated as valuable pieces of information.

- Bringing the conversation back to our agreed hypothesis provided a useful anchor and a clear point of reference while providing very rich data and a set of co-created research questions to be further explored.

- Overall, it was a challenging experience that made us grow collectively and enriched us personally by embracing other perspectives and interpretations. 
A4's goal for participation:

- To produce an inclusive assessment of a complex social setting and gain a deeper understanding of the kind of tensions and related usability challenges that might arise.

A4's key experiences:

- As an early career researcher, I admittedly found the notion of leading a focus group that consisted of far more experienced academics a bit daunting. It was therefore heartening to see the manner in which the immersive nature of our framework quickly pushed aside all hierarchies as everyone in the group engaged the tasks on hand as equals.

- Yet, it was not the prospect of gaining leadership skills that Photo of Researcher motivated me to take part in the project. By involving a highly diverse set of participants representing all major fields pertaining to mental healthcare, we essentially created a melting pot for diverse ideas and perspectives. As a designer seeking to gain a better understanding of diverse user contexts, this was an opportunity I simply could not pass up on. By subsequently transcribing and analysing recordings of our focus group sessions, I was given the opportunity to closely examine the key points of friction that arose and the distinct ways in which people related to them.

- Looking back, it has become increasingly apparent to me that academic disciplines are in fact largely man-made artificial constructs. When searching for answers to novel intellectual problems, we are often inadvertently limiting ourselves by adhering to such predefined discourses or perspectives. Yet, it is beyond these self-imposed limitations, that we tend to arrive at the most fascinating and ground breaking realisations. The framework in this sense turned out to serve as an interface between people with different backgrounds, catalysing a process of erosion and transcension of the artificial boundaries between established fields. Once the dust settled, we were able to "connect the dots" and gain a far more holistic understanding of problems and potential solutions than what would be possible through confinement to any one single discipline.

\section{Group Reflection}

We all gained new understandings of different disciplinary perspectives. We discovered through sustained communications that there was synthesis of knowledge and shifting in our thinking and mental models. A2's framework was key in aiding the process as it gave us a focus, the opportunity to explore key issues that were pertinent to all disciplines, ethics and design and use of digital technology. We all agreed, using the framework to structure the running of the focus group sessions during the workshop was novel. It enabled us to generate a rich set of data that not only met our individual disciplinary needs but also met the needs of our emergent interdisciplinary research project. We gained a shared understanding of the ethical dilemmas and concerns associated with the design and use of digital technology and of the data for creating the agents for creating social simulation modelling.

In line with the frequently sought after "inclusiveness" of research approaches (e.g. Nind et al., 2017), our framework was being tested in a new environment with a heterogeneous group of stakeholders. This resulted in a situation where our differing perspectives were brought to the fore as we began to grapple with different methodological approaches and terminologies. After spending a substantial amount of time analysing and debating the retrieved information, we eventually reached the conclusion that the potential usefulness of our original information-gathering framework exceeded mere development and calibration of simulation models. We realised that it had also evolved into a helpful tool for facilitating conversations and deliberations between members of distinct academic disciplines. 
These conversations and deliberations resulted in the production of outputs that went beyond the ones that would normally be produced if worked with our own disciplinary boundaries. Much like Teare and Taks (2019), our work thus highlights and illustrates the potential for distinct academic disciplines to develop a mutually complementary relationships. The novel insight we gained from our endeavours formed the basis for a paper that we jointly prepared for submission to a Human Computer Interaction conference (Perez et al., 2019), as well as other ongoing collaborations.

\section{Conclusion}

On reflection, we felt that we had inadvertently achieved a number of the key values and benefits of an interdisciplinary approach, namely, synthesis and integration of knowledge, collaborative working and shifting in thinking (Stember 1991, Carr et al 2018). We also realised that, unlike many of the examples in the literature, we had not approached what we were doing to try to establish how well an interdisciplinary approach works in practice nor were we aiming to go beyond the conventional definition. It was done with little or no conceptualisation that what we were doing was interdisciplinary research in its true meaning. Therefore, we had not framed what we were doing in the parameters of an interdisciplinary approach. In hindsight, we approached this endeavour of interdisciplinary working unencumbered by the usual associated expectations. We feel our experience does provide an additional perspective to the discourse around how interdisciplinarity is currently framed and conceptualised. Our knowledge integration, for example, was very much driven by our need to effectively communicate and understand each other's worldview and be respectful of each other (Stember 1997). We knew that in order for things to advance, we had to have a common language that we could all operate with. What helped us all was having a natural curiosity for each other's discipline which resulted in us conducting research that we feel crosses disciplinary boundaries.

\section{Acknowledgments}

Elvira Perez Vallejos acknowledges the resources of the NIHR Nottingham Biomedical Research Centre.

\section{References}

Aboebela S, Larson E, Bakken S, Olveen C, Formicola A, Sherry A G, Haas J, and Gebbie KM (2007) Defining interdisciplinary research: Conclusions from a critical review of the literature. Health Services Research, 42(1), 329-346.

Bonabeau E (2002) Agent-based modelling: Methods and techniques for simulating human systems. In: Proceedings of the National Academy of Sciences, 99(suppl3), 7280-7287.

Brown RR, Deletic A, and Wong TH (2015) How to catalyse collaboration: Turn the fraught flirtation between the social and biophysical sciences into fruitful partnerships with these five principles. Nature, 525(7569), 315-318.

Carr G, Loucks DP, and Bloschl G (2018) Gaining insights into interdisciplinary research and education programmes: A framework for evaluation. Research Policy, 47, 35-48.

Committee on Facilitating Interdisciplinary Research (2004) Facilitating Interdisciplinary Research. National Academies. Washington: National Academy Press.

Danermark B (2019) Applied interdisciplinary research: a critical realist perspective, Journal of Critical Realism, 18(4), 368-382.

Gleed, A, and Marchant, D (2016) Interdisciplinarity: survey report for the Global Research Council 2016. DJS Research. 
Goulden M, Greiffenhagen C, Crowcroft J, McAuley D, Mortier R, Radenkovic M, and Arjuna Sathiaseelan (2017) Wild interdisciplinarity: ethnography and computer science, International Journal of Social Research Methodology, 20(2), 137-150.

Huutoniemia K, Thompson Klein J, Bruun H, and Hukkinen J (2010) Analyzing interdisciplinarity: Typology and indicators. Research Policy, 39(1), 79-88.

Kolb DA (1984) Experiential Learning: Experience as the Source of Learning and Development. Englewood Cliffs, NJ: Prentice Hall.

Mallegowda P (2013) Interdisciplinary research: Way forward for biodiversity conservation. Current Science, 105(9), 1202-1203.

Nind, M, Armstrong, A, Cansdale, M, Collis, A, Hooper, C, Parsons, S, \& Power, A (2017) TimeBanking: towards a co-produced solution for power and money issues in inclusive research. International Journal of Social Research Methodology, 20(4), 387-400.

Perez Vallejos E, Nilsson T, Siebers PO, Siebert P, Craven M, and Fuentes C (2019) Untangling multistakeholder perspectives in digital mental healthcare. Computing and Mental Health. Symposium at CHI 2019. Available at http://mentalhealth.media.mit.edu/wpcontent/uploads/sites/15/2019/04/CMH2019_paper_11.pdf

Siebers PO and Klügl F (2017) What software engineering has to offer to agent-based social simulation. In: Edmonds B and Meyer R (Eds). Simulating Social Complexity: A Handbook - $2 e$. Berlin: Springer.

Stember M (1991) Advancing the social sciences through the interdisciplinary enterprise. The Social Science Journal, 28(1), 1-14.

Teare, G and Taks, M (2019) Extending the scoping review framework: a guide for interdisciplinary researchers. International Journal of Social Research Methodology, 1-5.

Van Teijlingen ER, Regmi PR, Adhikary P, Aryal N, and Simkhada P. (2019) Interdisciplinary research in public health: Not quite straightforward. Health Prospect. 18(1), 4-7.

Wikipedia (2019) Wikipedia: The Sims https://en.wikipedia.org/wiki/The Sims [last accessed 19/06/2019]. 